\title{
Effect of Titanium Oxide on Structure, Bearing Properties of Tin-Antimony-Lead and Tin-Aluminum Alloys
}

\author{
Abu Bakr El- Bediwi \\ Metal Physics Lab., Physics \\ Department, Faculty of Science, \\ Mansoura University \\ Mansoura, Egypt
}

\author{
Abbas Al- Bawee \\ Faculty of engineering, University of \\ Diayala \\ Diayala, Iraq
}

\author{
Mustafa Kamal \\ Metal Physics Lab., Physics \\ Department, Faculty of Science, \\ Mansoura University \\ Mansoura, Egypt
}

\begin{abstract}
Effect of adding titanium oxide nanoparticles $\left(\mathrm{TiO}_{2}\right)$ on structure, elastic moduli, Vickers hardness, internal friction, electrical resistivity and thermal properties of tin-antimony-lead and tin-aluminum bearing alloys have been investigated. Elastic modulus, Vickers hardness and thermal diffusivity of $\mathrm{Sn}_{87} \mathrm{Sb}_{10} \mathrm{~Pb}_{3}$ and $\mathrm{Sn}_{80} \mathrm{Al}_{20}$ alloys increased after adding $\mathrm{TiO}_{2}$ nanoparticles. Internal friction, thermal conductivity and specific heat of $\mathrm{Sn}_{87} \mathrm{Sb}_{10} \mathrm{~Pb}_{3}$ and $\mathrm{Sn}_{80} \mathrm{Al}_{20}$ alloys varied after adding $\mathrm{TiO}_{2}$ nanoparticles. Adding titanium oxide nanoparticles improved bearing properties, such as strengthens and internal friction of $\mathrm{Sn}_{87} \mathrm{Sb}_{10} \mathrm{~Pb}_{3}$ and $\mathrm{Sn}_{80} \mathrm{Al}_{20}$ alloys. The $\mathrm{Sn}_{85.5} \mathrm{Sb}_{10} \mathrm{~Pb}_{3}\left(\mathrm{TiO}_{2}\right)_{1.5}$ alloy has best properties for automotive industry. Also $\mathrm{Sn}_{78.5} \mathrm{Al}_{20}\left(\mathrm{TiO}_{2}\right)_{1.5}$ alloy has best properties for marine applications.
\end{abstract}

Key words: titanium oxide, internal friction, thermal properties, structure, hardness, resistivity, bearing alloys

\section{Introduction}

Bearings are used to prevent friction between parts during relative movement. In machinery they fall into two primary categories: anti-friction or rolling element bearings and hydrodynamic journal bearings. Today, the term Babbitt covers a collection of "white metal" alloys consisting generally of a tin or lead base accompanied by antimony and copper. Babbitt metal is used as the lining for bearing shells of cast iron, steel and bronze. Fry manufactures two basic types of babbitt, high-tin alloys and high-lead alloys. Both are relatively low melting materials consisting of hard compound in a soft matrix. Al-Sn alloys have a very long history (Forrester 1960) to be used as bearing materials [1]. These alloys provide a good combination of strength and surface properties [2]. The fatigue strength of cold worked and heat treated Al-20\%Sn$1 \% \mathrm{Cu}$ alloy having reticular structure is close to that of $\mathrm{Cu}-30 \% \mathrm{~Pb}$ alloy with higher seizure resistance [3]. Aluminium has a low modulus of elasticity and apart from indium, lead has the lowest modulus of elasticity of all the soft phases alloying with aluminium [4]. Al-Sn based alloys are widely used as sliding bearing materials in automobile and shipbuilding industry $[5,6]$. HVOF spray process has been introduced by McCartney to prepare Al-Sn-Si bearing alloy coatings. Post heat treatment of the HVOF sprayed coating at $300{ }^{\circ} \mathrm{C}$ proved the coarsening of tin and precipitate of $\mathrm{Si}$ in the coating [7, 8]. Based on the feasibility of preparing oxygen sensitive metal coatings, cold spray was also introduced to deposit Al-Sn binary alloy coatings [9]. Al-5Sn coating can be deposited by high pressure cold spray with nitrogen while Al-10Sn can only be deposited by low pressure cold spray with helium gas. Both $\mathrm{Al}-5 \mathrm{Sn}$ and $\mathrm{Al}-10 \mathrm{Sn}$ coatings present dense structures. The coarsening and/or migration of Sn phase in the coatings were observed when the annealing temperature exceeds $200{ }^{\circ} \mathrm{C}$. Furthermore, the microhardness of the coatings decreased significantly at the annealing temperature of $250{ }^{\circ} \mathrm{C}$ [10]. Aluminum tin and leaded aluminium alloys slightly differ in mechanical properties. Frictional states created during sliding against steel shaft under oil lubrication were not much different. Leaded aluminium alloy bushes show marginally lower friction than the conventional ones [11]. Adding $\mathrm{Cu} / \mathrm{Pb}$ to $\mathrm{Sn}-\mathrm{Sb}$ alloy improve their elastic modulus, internal friction, hardness and thermal conductivity [12]. The friction coefficients of $\mathrm{Sn}-20.2 \% \mathrm{Sb}-16.6 \% \mathrm{~Pb}-2.6 \% \mathrm{Cu}$ are lower than that of $\mathrm{Sn}-7.2 \% \mathrm{Sb}-0.4 \% \mathrm{~Pb}-3 \% \mathrm{Cu}$ under all scratch test conditions [13]. Structure, hardness, mechanical and electrical transport properties of $\mathrm{Sn}_{90-\mathrm{x}} \mathrm{Sb}_{10} \mathrm{Bi}_{\mathrm{x}}(\mathrm{x}=0$, or $\mathrm{x} \geq 1)$ alloys have been studied and analyzed [14]. The effects of solidification rate and microadditions on mechanical properties and micromorphology of $\mathrm{SnSb}_{10.4}$ alloy have been studied [15]. Creep behaviour, elastic modulus and internal friction of $\mathrm{SnSb}_{10} \mathrm{Cu}_{2} \mathrm{X}_{2}(\mathrm{X}=\mathrm{Pb}, \mathrm{Ag}, \mathrm{Se}, \mathrm{Cd}$ and $\mathrm{Zn})$ alloys have been investigated also stress exponent values have been determined using Mulhearn-Tabor method [16]. The aim of this research was to investigate the effect of adding titanium oxide nanoparticles $\left(\mathrm{TiO}_{2}\right)$ on structure, elastic moduli, Vickers hardness, internal friction and thermal properties of tin-antimony-lead and tinaluminum bearing alloys.

\section{Experimental work}

Two groups of quaternary bearing alloys, tin- antimonylead- titanium oxide and tin- aluminum- titanium oxide, were used. These groups' alloys were molten in the muffle furnace using (high purity more than $99.95 \%$ ) tin, antimony, lead, aluminum and titanium oxide. The resulting ingots were turned and re-melted several times to increase the homogeneity of the ingots. From these ingots, long ribbons of about 3-5 mm width and $\sim 70 \mu \mathrm{m}$ thickness were prepared as the test samples by directing a stream of molten alloy onto the outer surface of rapidly revolving copper roller with surface velocity $31 \mathrm{~m} / \mathrm{s}$ giving a cooling rate of $3.7 \times 10^{5} \mathrm{k} / \mathrm{s}$. The samples then cut into convenient shape for the measurements using double knife cuter. Structure of used alloys was performed using an Shimadzu X-ray Diffractometer (Dx-30, Japan) of $\mathrm{Cu}-\mathrm{K} \alpha$ radiation with $\lambda=1.54056 \AA$ at $45 \mathrm{kV}$ and $35 \mathrm{~mA}$ and $\mathrm{Ni}-$ filter in the angular range $2 \theta$ ranging from 0 to $100^{\circ}$ in continuous mode with a scan speed $5 \mathrm{deg} / \mathrm{min}$. Electrical resistivity of used alloys was measured by double bridge method. The melting endotherms of used alloys were obtained using a SDT Q600 V20.9 Build 20 instrument. A digital Vickers microhardness tester, (Model-FM-7- Japan), was used to measure Vickers hardness values of used alloys. $\mathrm{Q}^{-1}$, the elastic modulus $\mathrm{E}$ and 
thermal diffusivity $D_{\text {th }}$ of used alloys were determined using the dynamic resonance method [17-19].

$$
\begin{gathered}
\left(\frac{E}{\rho}\right)^{1 / 2}=\frac{2 \pi \mathbf{L}^{2} \mathbf{f}_{\mathrm{O}}}{k \mathbf{z}^{2}} \\
\mathbf{Q}^{-1}=0.5773 \frac{\Delta \mathbf{f}}{\mathbf{f}_{\mathrm{O}}} \\
D_{\mathrm{th}}=\frac{2 \mathbf{d}^{2} \mathbf{f}_{\mathrm{O}}}{\pi}
\end{gathered}
$$

Where $\rho$ is the density of the sample under test, $\mathrm{L}$ is the length of the vibrated part of the sample, $\mathrm{k}$ is the radius of gyration of cross section perpendicular to its plane of motion, $\mathrm{f}_{0}$ is the resonance frequency and $z$ is the constant depends on the mode of vibration and is equal to 1.8751. $\Delta \mathrm{f}$ is the half width of the resonance curve.

\section{Results and discussions}

Effect of adding $\mathrm{TiO}_{2}$ nanoparticles on structure of $\mathrm{Sn}-\mathrm{Sb}-\mathrm{Pb}$ alloy

X-ray diffraction patterns of $\mathrm{Sn}_{87-\mathrm{x}} \mathrm{Sb}_{10} \mathrm{~Pb}_{3}\left(\mathrm{TiO}_{2}\right)_{\mathrm{x}}(\mathrm{x}=0.5,1$ and $1.5 \mathrm{wt} . \%$ ) rapidly solidified alloys have lines corresponding to $\beta-\mathrm{Sn}, \mathrm{Pb} /$ or $\mathrm{Sb}$ and $\mathrm{SbSn}$ intermetallic phases as shown in Figure 1. $\mathrm{X}$-ray analysis show that, adding $\mathrm{TiO}_{2}$ to $\mathrm{Sn}_{87} \mathrm{Sb}_{10} \mathrm{~Pb}_{3}$ alloy caused a change in Sn matrix structure such as lattice parameters and formed crystal structure (crystallinity, crystal size and the orientation) as seen in Table 1( $\mathrm{a}$ and $\mathrm{b})$. That is because $\mathrm{TiO}_{2}$ nanoparticles dissolved in Sn matrix formed a solid solution and other accumulated particles formed a traces of phases.
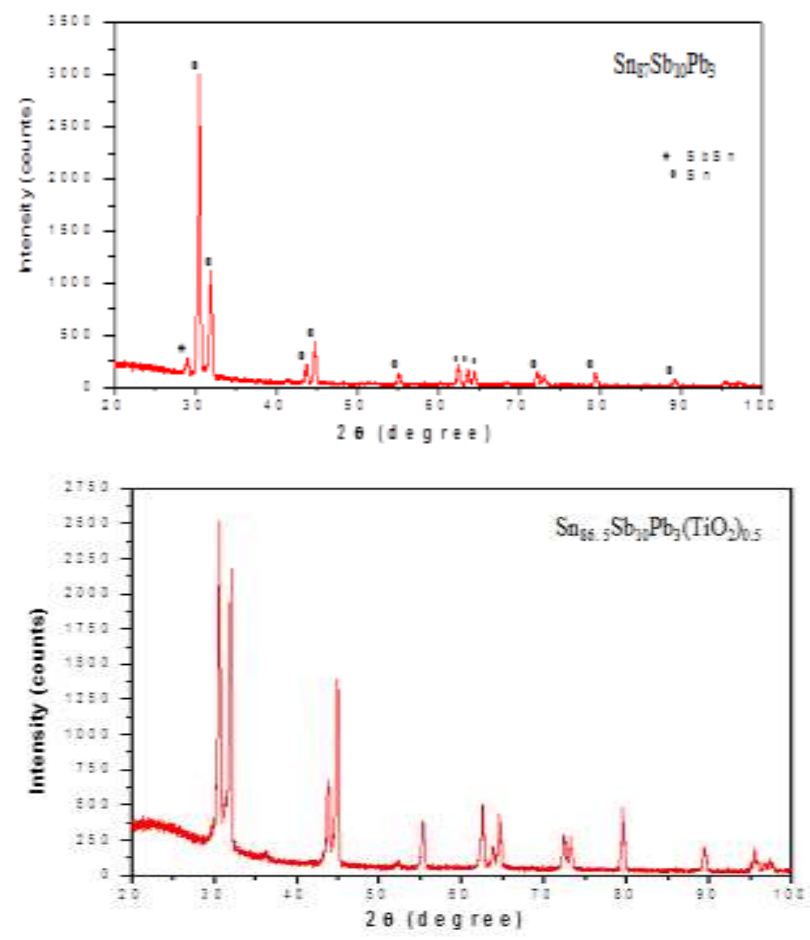
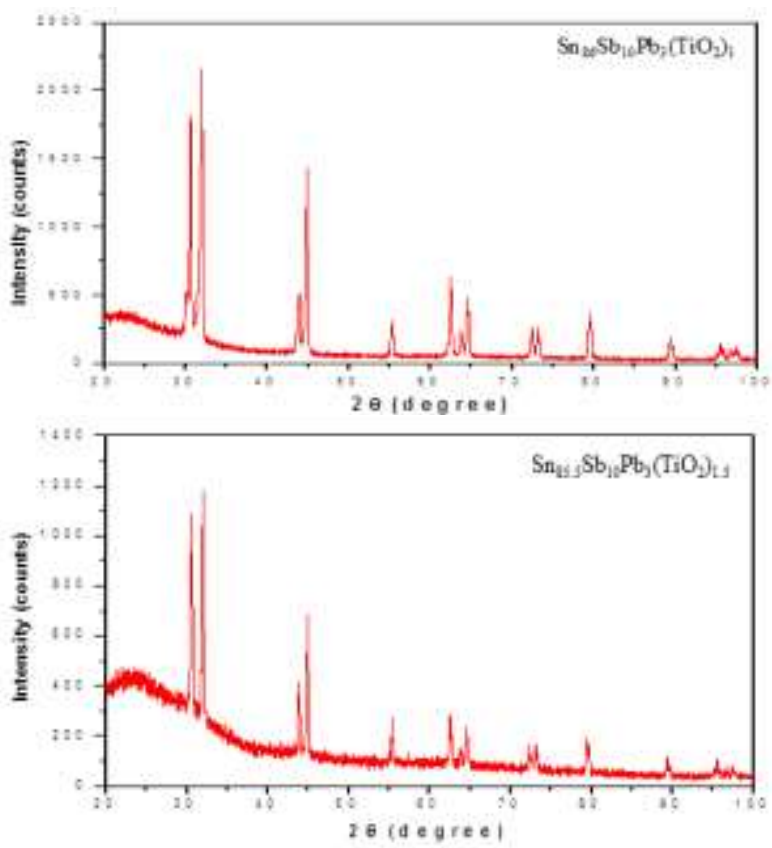

Figure 1:- x-ray diffraction patterns of $\mathrm{Sn}_{87-\mathrm{x}} \mathrm{Sb}_{10} \mathrm{~Pb}_{3}\left(\mathrm{TiO}_{2}\right)_{\mathrm{x}}$ alloys

Table 1a:- $\mathrm{x}$-ray diffraction analysis of $\mathrm{Sn}_{87-\mathrm{x}} \mathrm{Sb}_{10} \mathrm{~Pb}_{3}\left(\mathrm{TiO}_{2}\right)_{\mathrm{x}}$ alloys

\begin{tabular}{|c|c|c|c|c|c|}
\hline \multicolumn{7}{|c|}{$\mathrm{Sn}_{87} \mathrm{Sb}_{10} \mathrm{~Pb}_{3}$} \\
\hline $2 \theta$ & $\mathrm{d} \AA$ & $\mathrm{Int} . \%$ & $\mathrm{FWHM}$ & Phase & hkl \\
\hline 29.0695 & 3.07186 & 4.37 & 0.2755 & $\mathrm{SbSn}$ & 101 \\
\hline 30.4985 & 2.92868 & 100 & 0.2400 & $\mathrm{Sn}$ & 200 \\
\hline 30.6095 & 2.92557 & 69.41 & 0.0720 & $\mathrm{Sn}$ & 200 \\
\hline 31.9779 & 2.796439 & 34.11 & 0.1920 & $\mathrm{Sn}$ & 101 \\
\hline 41.5347 & 2.17246 & 0.77 & 0.5760 & $\mathrm{SbSn}$ & 012 \\
\hline 43.6580 & 2.07160 & 5.13 & 0.4320 & $\mathrm{Sn}$ & 220 \\
\hline 44.7618 & 2.02304 & 12.29 & 0.3360 & $\mathrm{Sn}$ & 211 \\
\hline 55.1013 & 1.66539 & 3.75 & 0.3360 & $\mathrm{Sn}$ & 301 \\
\hline 62.4237 & 1.48648 & 5.81 & 0.3360 & $\mathrm{Sn}$ & 112 \\
\hline 63.6354 & 1.46106 & 4.05 & 0.3840 & $\mathrm{Sn}$ & 400 \\
\hline 64.3731 & 1.44609 & 3.59 & 0.4320 & $\mathrm{Sn}$ & 321 \\
\hline 68.2871 & 1.37243 & 0.41 & 0.5760 & $\mathrm{SbSn}$ & 113 \\
\hline 72.1128 & 1.30874 & 3.63 & 0.2880 & $\mathrm{Sn}$ & 420 \\
\hline 72.9123 & 1.29635 & 2.75 & 0.2880 & $\mathrm{Sn}$ & 411 \\
\hline 79.3161 & 1.20698 & 3.59 & 0.2880 & $\mathrm{Sn}$ & 321 \\
\hline 89.0610 & 1.09840 & 2.05 & 0.2400 & $\mathrm{Sn}$ & 432 \\
\hline 95.3295 & 1.04205 & 1.46 & 0.3840 & $\mathrm{Sn}$ & 103 \\
\hline 96.3177 & 1.03396 & 0.64 & 0.4800 & $\mathrm{Sn}$ & 330 \\
\hline 97.0846 & 1.02783 & 0.75 & 0.4800 & $\mathrm{Sn}$ & 521 \\
\hline
\end{tabular}


International Journal of Science and Engineering Applications

Volume 4 Issue 2, 2015, ISSN-2319-7560 (Online)

\begin{tabular}{|c|c|c|c|c|c|}
\hline 89.3181 & 1.09682 & 7.16 & 0.1771 & Sn & 432 \\
\hline 95.4954 & 1.04154 & 7.27 & 0.1771 & Sn & 103 \\
\hline 97.4966 & 1.02458 & 2.88 & 0.576 & Sn & 521 \\
\hline
\end{tabular}

\begin{tabular}{|c|c|c|c|c|c|}
\hline \multicolumn{7}{|c|}{$\mathrm{Sn}_{86.5} \mathrm{Sb}_{10} \mathrm{~Pb}_{3}\left(\mathrm{TiO}_{2}\right)_{0.5}$} \\
\hline $2 \theta$ & $\mathrm{d} \AA$ & $\mathrm{Int} \%$ & $\mathrm{FWHM}$ & Phase & hkl \\
\hline 30.6118 & 2.92052 & 100 & 0.2558 & $\mathrm{Sn}$ & 200 \\
\hline 32.0305 & 2.79433 & 80.39 & 0.2558 & $\mathrm{Sn}$ & 101 \\
\hline 36.1764 & 2.48304 & 1.74 & 0.4723 & $\mathrm{~Pb}$ & 220 \\
\hline 43.7948 & 2.06715 & 25.53 & 0.3346 & $\mathrm{Sn}$ & 220 \\
\hline 44.8366 & 2.02152 & 56.58 & 0.2952 & $\mathrm{Sn}$ & 211 \\
\hline 52.2999 & 1.74926 & 1.68 & 0.3936 & $\mathrm{SbSn}$ & 021 \\
\hline 55.2681 & 1.66214 & 13.94 & 0.2755 & $\mathrm{Sn}$ & 301 \\
\hline 62.4854 & 1.48639 & 18.62 & 0.2558 & $\mathrm{Sn}$ & 112 \\
\hline 63.759 & 1.45974 & 5.28 & 0.2755 & $\mathrm{Sb}$ & 107 \\
\hline 64.5448 & 1.44385 & 16.61 & 0.2165 & $\mathrm{Sn}$ & 321 \\
\hline 72.3498 & 1.30611 & 10.53 & 0.2165 & $\mathrm{Sb}$ & 018 \\
\hline 73.1307 & 1.29409 & 8.95 & 0.2558 & $\mathrm{Sn}$ & 411 \\
\hline 79.4279 & 1.20656 & 16.98 & 0.1968 & $\mathrm{Sn}$ & 321 \\
\hline 89.2927 & 1.09706 & 6.94 & 0.1968 & $\mathrm{Sn}$ & 432 \\
\hline 95.4951 & 1.04154 & 6.04 & 0.1968 & $\mathrm{Sn}$ & 103 \\
\hline 96.6061 & 1.0325 & 1.82 & 0.2362 & $\mathrm{Sn}$ & 440 \\
\hline 97.2882 & 1.02622 & 3.09 & 0.24 & $\mathrm{Sn}$ & 521 \\
\hline
\end{tabular}

\begin{tabular}{|c|c|c|c|c|c|}
\hline \multicolumn{7}{|c|}{$\mathrm{Sn}_{86} \mathrm{Sb}_{10} \mathrm{~Pb}_{3}\left(\mathrm{TiO}_{2}\right)_{1}$} \\
\hline $2 \theta$ & $\mathrm{d} \AA$ & $\mathrm{Int} . \%$ & FWHM & Phase & hkl \\
\hline 30.6133 & 2.92038 & 86.52 & 0.2558 & $\mathrm{Sn}$ & 200 \\
\hline 32.0016 & 2.79679 & 100 & 0.2558 & $\mathrm{Sn}$ & 101 \\
\hline 43.8373 & 2.06525 & 22.17 & 0.2755 & $\mathrm{Sn}$ & 220 \\
\hline 44.8304 & 2.02178 & 66.02 & 0.2362 & $\mathrm{Sn}$ & 211 \\
\hline 55.2842 & 1.66169 & 12.86 & 0.3346 & $\mathrm{Sn}$ & 301 \\
\hline 62.4583 & 1.48697 & 25.71 & 0.2558 & $\mathrm{Sn}$ & 112 \\
\hline 63.7276 & 1.46038 & 7.61 & 0.2165 & $\mathrm{Sb}$ & 107 \\
\hline 64.4749 & 1.44525 & 17.75 & 0.2165 & $\mathrm{Sn}$ & 321 \\
\hline 72.3608 & 1.30594 & 10.42 & 0.1771 & $\mathrm{Sb}$ & 018 \\
\hline 73.0899 & 1.29471 & 10.52 & 0.1771 & $\mathrm{Sn}$ & 411 \\
\hline 79.4307 & 1.20552 & 15.71 & 0.264 & $\mathrm{Sn}$ & 321 \\
\hline 79.7468 & 1.20453 & 8.87 & 0.192 & $\mathrm{Sn}$ & 321 \\
\hline 89.3151 & 1.09594 & 7.47 & 0.264 & $\mathrm{Sn}$ & 432 \\
\hline 89.6708 & 1.09523 & 4.46 & 0.192 & $\mathrm{Sn}$ & 431 \\
\hline 95.0358 & 1.04449 & 1.75 & 0.24 & $\mathrm{Sn}$ & 103 \\
\hline 95.5155 & 1.04051 & 5.28 & 0.216 & $\mathrm{Sn}$ & 103 \\
\hline 95.8764 & 1.04013 & 3.21 & 0.216 & $\mathrm{Sn}$ & 332 \\
\hline 96.6134 & 1.03158 & 3.09 & 0.24 & $\mathrm{Sn}$ & 440 \\
\hline 97.3745 & 1.02554 & 4.55 & 0.24 & $\mathrm{Sn}$ & 521 \\
\hline
\end{tabular}

Table 1b:- lattice parameters and crystal size of $\beta$-Sn in $\mathrm{Sn}_{87-\mathrm{x}} \mathrm{Sb}_{10} \mathrm{~Pb}_{3}\left(\mathrm{TiO}_{2}\right)_{\mathrm{x}}$ alloys

\begin{tabular}{|c|c|c|c|c|}
\hline Alloys & a $\AA$ & c $\AA$ & $\mathrm{V} \AA^{3}$ & $\tau \AA$ \\
\hline $\mathrm{Sn}_{87} \mathrm{Sb}_{10} \mathrm{~Pb}_{3}$ & 5.857 & 3.174 & 108.883 & 355.44 \\
\hline $\mathrm{Sn}_{86 .} \mathrm{Sb}_{10} \mathrm{~Pb}_{3}\left(\mathrm{TiO}_{2}\right)_{0.5}$ & 5.841 & 3.18 & 108.62 & 514.283 \\
\hline $\mathrm{Sn}_{86} \mathrm{Sb}_{10} \mathrm{~Pb}_{3}\left(\mathrm{TiO}_{2}\right)_{1}$ & 5.841 & 3.2 & 109.15 & 508.035 \\
\hline $\mathrm{Sn}_{85.5} \mathrm{Sb}_{10} \mathrm{~Pb}_{3}\left(\mathrm{TiO}_{2}\right)_{1.5}$ & 5.843 & 3.19 & 108.83 & 529.678 \\
\hline
\end{tabular}

Scanning electron micrographs, SEM, of $\mathrm{Sn}_{87-}$ ${ }_{x} \mathrm{Sb}_{10} \mathrm{~Pb}_{3}\left(\mathrm{TiO}_{2}\right)_{\mathrm{x}}(\mathrm{x}=0$ and 1.5 wt. \%) alloys show heterogeneity structure as shown in Figure 2. SEM micrographs of $\mathrm{Sn}_{87}$ ${ }_{x} \mathrm{Sb}_{10} \mathrm{~Pb}_{3}\left(\mathrm{TiO}_{2}\right)_{\mathrm{x}}$ alloys show $\beta$ - Sn matrix and other accumulated particles formed traces of phases and that is agreed with $\mathrm{x}$-ray results.
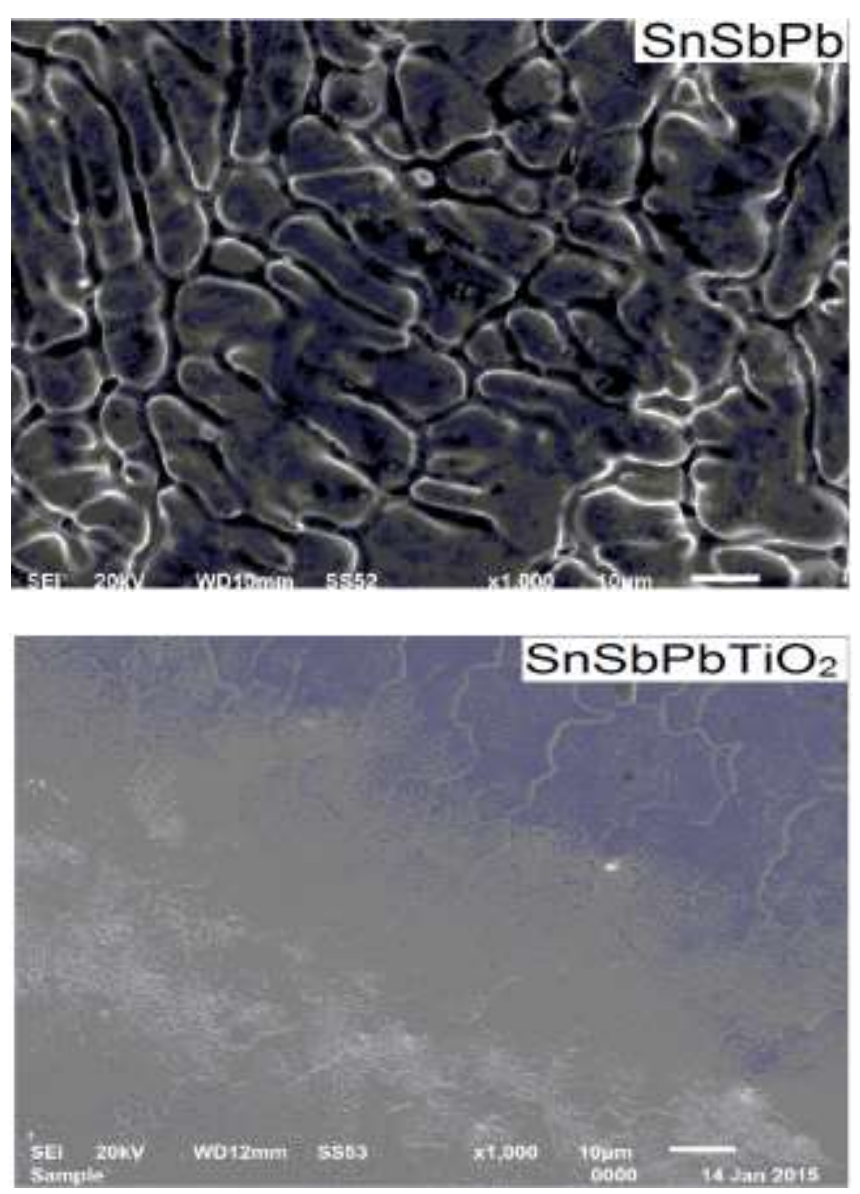

Figure 2:- $\mathrm{SEM}$ of $\mathrm{Sn}_{87} \mathrm{Sb}_{10} \mathrm{~Pb}_{3}$ and $\mathrm{Sn}_{85.5} \mathrm{Sb}_{10} \mathrm{~Pb}_{3}\left(\mathrm{TiO}_{2}\right)_{1.5}$ alloys 


\section{Effect of adding $\mathrm{TiO}_{2}$ nanoparticles on mechanical properties of Sn-Sb-Pb alloy}

The elastic constants are directly related to atomic bonding and structure. Elastic modului of $\mathrm{Sn}_{87-\mathrm{x}} \mathrm{Sb}_{10} \mathrm{~Pb}_{3}\left(\mathrm{TiO}_{2}\right)_{\mathrm{x}}$ alloys are listed in Table 2. Elastic modulus of $\mathrm{Sn}_{87} \mathrm{Sb}_{10} \mathrm{~Pb}_{3}$ alloy increased after adding different ratio from $\mathrm{TiO}_{2}$ nanoparticles. The $\mathrm{Sn}_{85.5} \mathrm{Sb}_{10} \mathrm{~Pb}_{3}\left(\mathrm{TiO}_{2}\right)_{1.5}$ alloy has highest elastic modulus.

Table 2:- elastic modului, internal friction and thermal diffusivity of $\mathrm{Sn}_{87-\mathrm{x}} \mathrm{Sb}_{10} \mathrm{~Pb}_{3}\left(\mathrm{TiO}_{2}\right)_{\mathrm{x}}$ alloys

\begin{tabular}{|c|c|c|c|c|c|}
\hline Alloys & $\begin{array}{c}\mathrm{E} \\
\mathrm{GPa}\end{array}$ & $\begin{array}{c}\mu \\
\mathrm{GPa}\end{array}$ & $\begin{array}{c}\mathrm{B} \\
\mathrm{GPa}\end{array}$ & $\mathrm{Q}^{-1}$ & $\begin{array}{c}\mathrm{D}_{\text {th }} \times 10^{-8} \\
\mathrm{~m}^{2} \text { Isec }\end{array}$ \\
\hline $\mathrm{Sn}_{87} \mathrm{Sb}_{10} \mathrm{~Pb}_{3}$ & 33.02 & 12.15 & 39.15 & 0.025 & 9.43 \\
\hline $\mathrm{Sn}_{86.5} \mathrm{Sb}_{10} \mathrm{~Pb}_{3}\left(\mathrm{TiO}_{2}\right)_{0.5}$ & 38.3 & 14.1 & 45.3 & 0.031 & 27.36 \\
\hline $\mathrm{Sn}_{86} \mathrm{Sb}_{10} \mathrm{~Pb}_{3}\left(\mathrm{TiO}_{2}\right)_{1}$ & 39.1 & 14.4 & 46.1 & 0.024 & 21.43 \\
\hline $\mathrm{Sn}_{85 .} \mathrm{Sb}_{10} \mathrm{~Pb}_{3}\left(\mathrm{TiO}_{2}\right)_{1.5}$ & 47.2 & 17.4 & 55.4 & 0.018 & 41.87 \\
\hline
\end{tabular}

The resonance curves $\mathrm{Sn}_{87-\mathrm{x}} \mathrm{Sb}_{10} \mathrm{~Pb}_{3}\left(\mathrm{TiO}_{2}\right)_{\mathrm{x}}$ alloys are shown in Figure 3. Calculated internal friction and thermal diffusivity of $\mathrm{Sn}_{87-\mathrm{x}} \mathrm{Sb}_{10} \mathrm{~Pb}_{3}\left(\mathrm{TiO}_{2}\right)_{\mathrm{x}}$ alloys are listed in Table 2 . Internal friction of $\mathrm{Sn}_{87} \mathrm{Sb}_{10} \mathrm{~Pb}_{3}$ alloy varied after adding different ratio from $\mathrm{TiO}_{2}$ nanoparticles. The $\mathrm{Sn}_{85.5} \mathrm{Sb}_{10} \mathrm{~Pb}_{3}\left(\mathrm{TiO}_{2}\right)_{1.5}$ alloy has lowest internal friction.

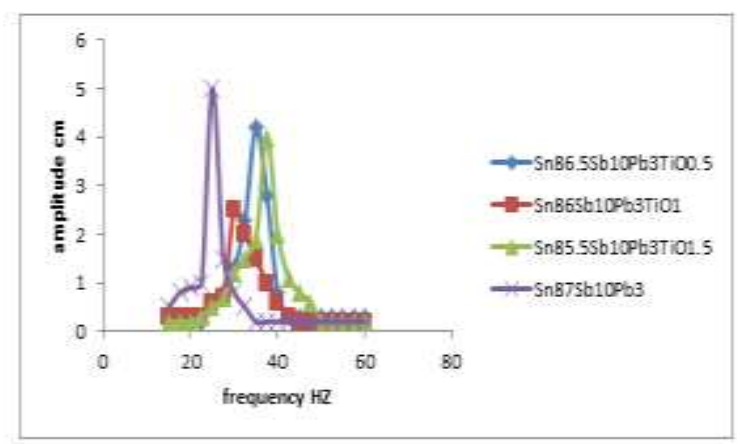

Figure 3:- resonance curves of $\mathrm{Sn}_{87-\mathrm{x}} \mathrm{Sb}_{10} \mathrm{~Pb}_{3}\left(\mathrm{TiO}_{2}\right)_{\mathrm{x}}$ alloys

The hardness is the property of material, which gives it the ability to resist being permanently deformed when a load is applied. Vickers hardness of $\mathrm{Sn}_{87-\mathrm{x}} \mathrm{Sb}_{10} \mathrm{~Pb}_{3}\left(\mathrm{TiO}_{2}\right)_{\mathrm{x}}$ alloys at 10 gram force and indentation time $5 \mathrm{sec}$ are shown in Table 3 . The minimum shear stress $\left(\tau_{\mathrm{m}}\right)$ value of $\mathrm{Sn}_{87-\mathrm{x}} \mathrm{Sb}_{10} \mathrm{~Pb}_{3}\left(\mathrm{TiO}_{2}\right)_{\mathrm{x}}$ alloys was calculated using the equation [9], where $v$ is Poisson's ratio of the elements in the alloy and then listed in Table 3.

$$
\tau_{m}=\frac{1}{2} H_{v}\left\{\frac{1}{2}(1-2 v)+\frac{2}{9}(1+v)[2(1+v)]^{\frac{1}{2}}\right\}
$$

Vickers hardness of $\mathrm{Sn}_{87} \mathrm{Sb}_{10} \mathrm{~Pb}_{3}$ alloy increased after adding $\mathrm{TiO}_{2}$ nanoparticles. That is because $\mathrm{TiO}_{2}$ dissolved in $\mathrm{Sn}$ matrix formed a hard inclusion increased its hardness. $\mathrm{Sn}_{85.5} \mathrm{Sb}_{10} \mathrm{~Pb}_{3}\left(\mathrm{TiO}_{2}\right)_{1.5}$ alloy has highest hardness.
Table 3:- Vickers hardness and minimum shear stress of $\mathrm{Sn}_{87-\mathrm{x}} \mathrm{Sb}_{10} \mathrm{~Pb}_{3}\left(\mathrm{TiO}_{2}\right)_{\mathrm{x}}$ alloys

\begin{tabular}{|c|c|c|}
\hline Alloys & $\mathrm{H}_{\mathrm{v}} \mathrm{kg} / \mathrm{mm}^{2}$ & $\mu_{\mathrm{s}} \mathrm{kg} / \mathrm{mm}^{2}$ \\
\hline $\mathrm{Sn}_{87} \mathrm{Sb}_{10} \mathrm{~Pb}_{3}$ & $28.52 \pm 1.8$ & 9.41 \\
\hline $\mathrm{Sn}_{86.5} \mathrm{Sb}_{10} \mathrm{~Pb}_{3}\left(\mathrm{TiO}_{2}\right)_{0.5}$ & $31.68 \pm 2.7$ & 10.45 \\
\hline $\mathrm{Sn}_{86} \mathrm{Sb}_{10} \mathrm{~Pb}_{3}\left(\mathrm{TiO}_{2}\right)_{1}$ & $36.53 \pm 3.3$ & 12.05 \\
\hline $\mathrm{Sn}_{85.5} \mathrm{Sb}_{10} \mathrm{~Pb}_{3}\left(\mathrm{TiO}_{2}\right)_{1.5}$ & $38.83 \pm 2.4$ & 12.81 \\
\hline
\end{tabular}

Effect of adding $\mathrm{TiO}_{2}$ on thermal properties and electrical resistivity of $\mathrm{Sn}-\mathrm{Sb}-\mathrm{Pb}$ alloy

Thermal analysis is often used to study solid state transformations as well as solid-liquid reactions. Figure 4 shows DSC thermographs for $\mathrm{Sn}_{87-\mathrm{x}} \mathrm{Sb}_{10} \mathrm{~Pb}_{3}\left(\mathrm{TiO}_{2}\right)_{\mathrm{x}}$ alloys. Little variation occurred in exothermal peak of $\mathrm{Sn}_{87} \mathrm{Sb}_{10} \mathrm{~Pb}_{3}$ alloy. The melting temperature and other thermal properties of $\mathrm{Sn}_{87-\mathrm{x}} \mathrm{Sb}_{10} \mathrm{~Pb}_{3}\left(\mathrm{TiO}_{2}\right)_{\mathrm{x}}$ alloys are listed in Table 4. Melting temperature of $\mathrm{Sn}_{87} \mathrm{Sb}_{10} \mathrm{~Pb}_{3}$ alloy decreased after adding $\mathrm{TiO}_{2}$ nanoparticles.

Crystalline defects serve as scattering center for conduction electrons in metals, so the increase in their number raises the imperfection. Electrical resistivity and calculated thermal conductivities of $\mathrm{Sn}_{87-\mathrm{x}} \mathrm{Sb}_{10} \mathrm{~Pb}_{3}\left(\mathrm{TiO}_{2}\right)_{\mathrm{x}}$ alloys are shown in Table 4. Electrical resistivity of $\mathrm{Sn}_{87} \mathrm{Sb}_{10} \mathrm{~Pb}_{3}$ alloy varied after adding $\mathrm{TiO}_{2}$ nanoparticles. That is because $\mathrm{TiO}_{2}$ nanoparticles dissolved in the $\mathrm{Sn}$ matrix playing as scattering center for conduction electrons caused a change in matrix structure.

Table 4:- electric resistivity and other thermal properties of $\mathrm{Sn}_{87-\mathrm{x}} \mathrm{Sb}_{10} \mathrm{~Pb}_{3}\left(\mathrm{TiO}_{2}\right)_{\mathrm{x}}$ alloys

\begin{tabular}{|c|c|c|c|c|}
\hline Alloys & $\begin{array}{c}\rho \times 10^{-6} \\
\Omega . c m\end{array}$ & $\begin{array}{l}\mathrm{K} \mathrm{W} \\
\mathrm{m}^{-1} \mathrm{~K}^{-1}\end{array}$ & $\begin{array}{l}\mathrm{C}_{\mathrm{p}} \times 10^{3} \\
\mathrm{~J} / \mathrm{kg} . \mathrm{k}\end{array}$ & $\begin{array}{l}\text { Melting } \\
\text { point }{ }^{\circ} \mathrm{C}\end{array}$ \\
\hline $\mathrm{Sn}_{87} \mathrm{Sb}_{10} \mathrm{~Pb}_{3}$ & 67.3 & 2.30 & 1.84 & 236.87 \\
\hline $\mathrm{Sn}_{86.5} \mathrm{Sb}_{10} \mathrm{~Pb}_{3}\left(\mathrm{TiO}_{2}\right)_{0.5}$ & 59.04 & 2.55 & 1.88 & 223.38 \\
\hline $\mathrm{Sn}_{86} \mathrm{Sb}_{10} \mathrm{~Pb}_{3}\left(\mathrm{TiO}_{2}\right)_{1}$ & 65.19 & 2.32 & 3.93 & 229.52 \\
\hline $\mathrm{Sn}_{85 .} \mathrm{Sb}_{10} \mathrm{~Pb}_{3}\left(\mathrm{TiO}_{2}\right)_{1.5}$ & 77.78 & 1.94 & 3.29 & 229.33 \\
\hline
\end{tabular}

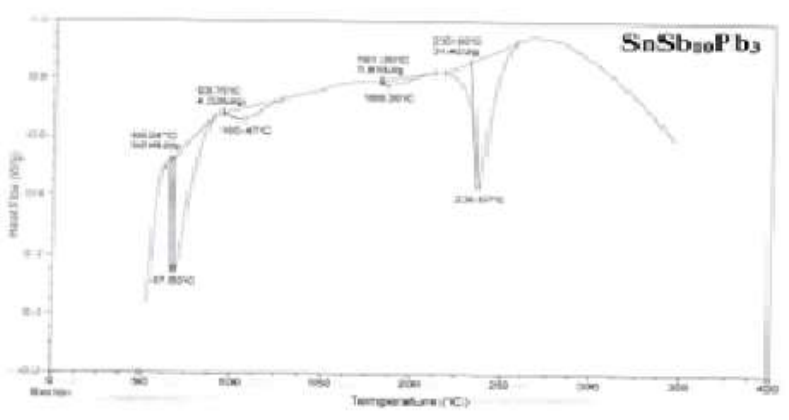



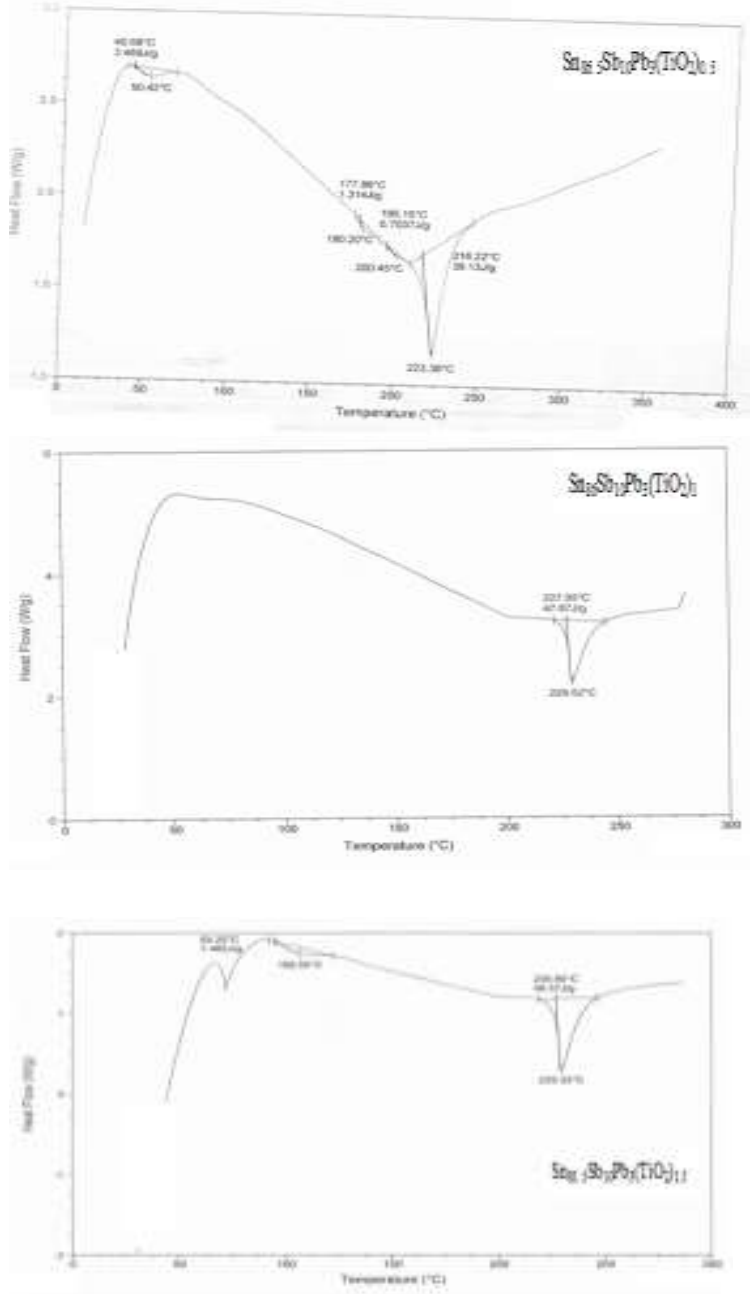

Figure 4:- DSC of $\mathrm{Sn}_{87-\mathrm{x}} \mathrm{Sb}_{10} \mathrm{~Pb}_{3}\left(\mathrm{TiO}_{2}\right)_{\mathrm{x}}$ alloys

\section{Effect of adding $\mathrm{TiO}_{2}$ nanoparticles on structure of $\mathrm{Sn}-\mathrm{Al}$ alloy}

$\mathrm{X}$-ray diffraction patterns of $\mathrm{Sn}_{80-\mathrm{x}} \mathrm{Al}_{20}\left(\mathrm{TiO}_{2}\right)_{\mathrm{x}}(\mathrm{x}=0.5,1$ and 1.5 wt. \%) alloys have lines corresponding to $\beta$ - $\mathrm{Sn}$ and $\mathrm{Al}$ phases as shown in Figure 5. X-ray analysis show that, adding $\mathrm{TiO}_{2}$ to $\mathrm{Sn}_{80} \mathrm{Al}_{20}$ alloy caused a change in $\mathrm{Sn}$ matrix such as lattice parameters and formed crystal structure (crystallinity, crystal size and the orientation) as seen in Table 5 ( $a$ and b). That is because $\mathrm{TiO}_{2}$ nanoparticles dissolved in $\mathrm{Sn}$ matrix formed a solid solution and other accumulated particles formed a traces of phases.
Scanning electron micrographs, $\mathrm{SEM}$, of $\mathrm{Sn}_{80-\mathrm{x}} \mathrm{Al}_{20}\left(\mathrm{TiO}_{2}\right)_{\mathrm{x}}$ alloys show heterogeneity structure as shown in Figure 6. SEM micrographs of $\mathrm{Sn}_{80-\mathrm{x}} \mathrm{Al}_{20}\left(\mathrm{TiO}_{2}\right)_{\mathrm{x}}$ alloys show $\beta$ - $\mathrm{Sn}$ matrix with other accumulated particles formed traces of phases and that agree with $\mathrm{x}$ ray results
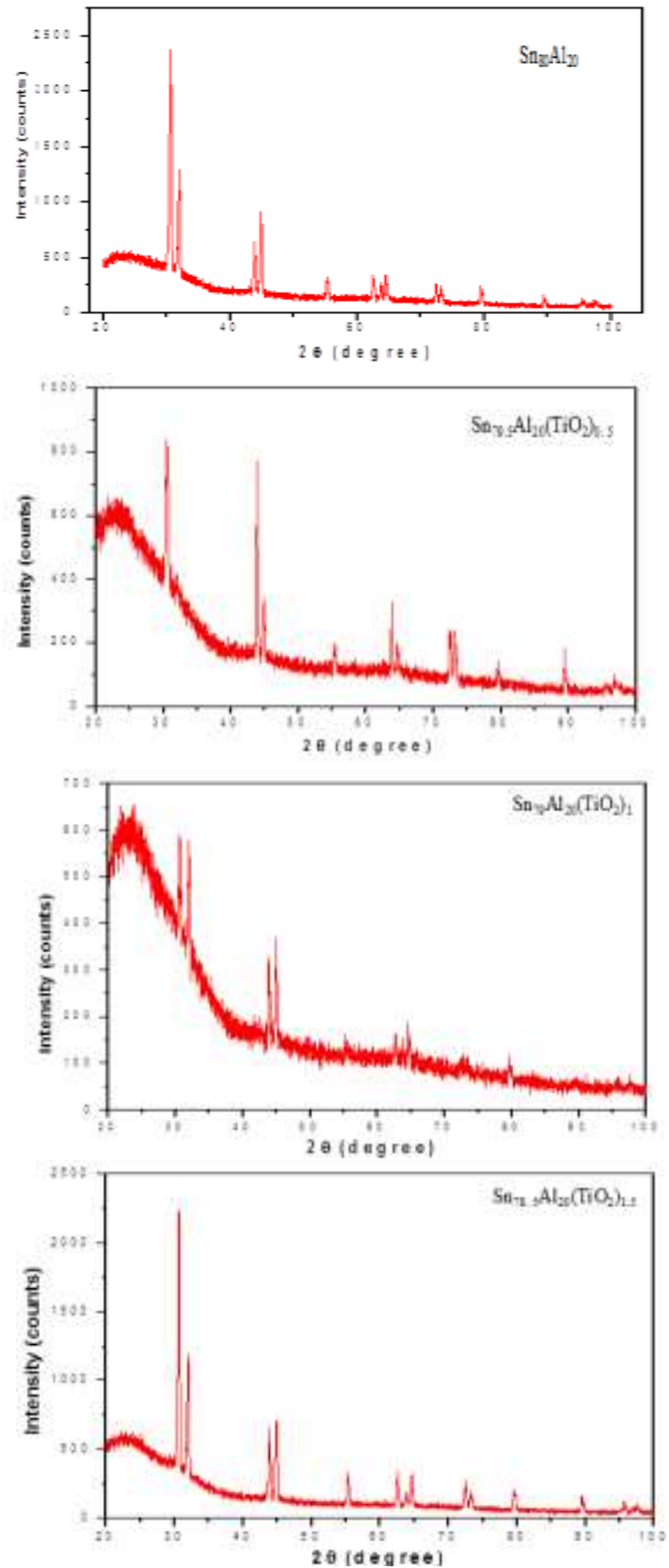

Figure 5:- $\mathrm{x}$-ray diffraction patterns of $\mathrm{Sn}_{80-\mathrm{x}} \mathrm{Al}_{20}\left(\mathrm{TiO}_{2}\right)_{\mathrm{x}}$ alloys 
International Journal of Science and Engineering Applications

Volume 4 Issue 2, 2015, ISSN-2319-7560 (Online)

\begin{tabular}{|c|c|c|c|c|c|}
\hline 73.45 & 1.28925 & 6.09 & 0.09 & Sn & 411 \\
\hline 79.7057 & 1.20206 & 11.97 & 0.576 & Sn & 321 \\
\hline 89.61 & 1.094 & 4.68 & 0.09 & Sn & 432 \\
\hline 95.67 & 1.0401 & 3.28 & 0.09 & Sn & 103 \\
\hline 97.69 & 1.02391 & 7.02 & 0.09 & Sn & 521 \\
\hline
\end{tabular}

Table 5a:- $\mathrm{x}$-ray diffraction analysis of $\mathrm{Sn}_{80-\mathrm{x}} \mathrm{Al}_{20}\left(\mathrm{TiO}_{2}\right)_{\mathrm{x}}$ alloys

\begin{tabular}{|c|c|c|c|c|c|}
\hline \multicolumn{7}{|c|}{$\mathrm{Sn}_{80} \mathrm{Al}_{20}$} \\
\hline $2 \theta$ & $\mathrm{d} \AA$ & Int. $\%$ & FWHM & Phase & hkl \\
\hline 30.5888 & 2.92025 & 100 & 0.12 & $\mathrm{Sn}$ & 200 \\
\hline 30.6656 & 2.92035 & 84.96 & 0.096 & $\mathrm{Sn}$ & 200 \\
\hline 31.9935 & 2.79516 & 46.62 & 0.216 & $\mathrm{Sn}$ & 101 \\
\hline 43.7803 & 2.0661 & 22.91 & 0.168 & $\mathrm{Sn}$ & 220 \\
\hline 44.8271 & 2.02025 & 33.94 & 0.12 & $\mathrm{Al}$ & 200 \\
\hline 55.2821 & 1.66037 & 9.25 & 0.432 & $\mathrm{Sn}$ & 301 \\
\hline 62.448 & 1.48596 & 9.2 & 0.24 & $\mathrm{Sn}$ & 112 \\
\hline 63.7441 & 1.45883 & 6.57 & 0.288 & $\mathrm{Sn}$ & 400 \\
\hline 64.4962 & 1.44363 & 10.04 & 0.24 & $\mathrm{Al} / \mathrm{Sn}$ & $220 / 321$ \\
\hline 72.3591 & 1.30489 & 7.2 & 0.192 & $\mathrm{Sn}$ & 411 \\
\hline 73.0968 & 1.29353 & 6.01 & 0.336 & $\mathrm{Sn}$ & 411 \\
\hline 79.4138 & 1.20574 & 7.3 & 0.24 & $\mathrm{Sn}$ & 312 \\
\hline 89.3336 & 1.09576 & 4.52 & 0.192 & $\mathrm{Sn}$ & 431 \\
\hline 95.6292 & 1.03957 & 1.42 & 0.768 & $\mathrm{Sn}$ & 103 \\
\hline 97.3885 & 1.02543 & 2.57 & 0.288 & $\mathrm{Sn}$ & 521 \\
\hline
\end{tabular}

\begin{tabular}{|c|c|c|c|c|c|}
\hline \multicolumn{7}{|c|}{$\mathrm{Sn}_{78.5} \mathrm{Al}_{20}\left(\mathrm{TiO}_{2}\right)_{1.5}$} \\
\hline $2 \theta$ & $\AA d$ & Int.\% & FWHM & Phase & hkl \\
\hline 30.6183 & 2.91991 & 100 & 0.2558 & $\mathrm{Sn}$ & 200 \\
\hline 32.0071 & 2.79632 & 45.14 & 0.2362 & $\mathrm{Sn}$ & 101 \\
\hline 43.9365 & 2.06082 & 23.02 & 0.2558 & $\mathrm{Sn}$ & 220 \\
\hline 44.8952 & 2.01901 & 30.74 & 0.2952 & $\mathrm{Al}$ & 211 \\
\hline 55.3468 & 1.65996 & 10.96 & 0.2558 & $\mathrm{Sn}$ & 301 \\
\hline 62.5078 & 1.48591 & 11.37 & 0.2362 & $\mathrm{Sn}$ & 112 \\
\hline 63.8614 & 1.45764 & 4.34 & 0.1968 & $\mathrm{Sb}$ & 107 \\
\hline 64.6256 & 1.44224 & 10.87 & 0.2165 & $\mathrm{Al} / \mathrm{Sn}$ & $220 / 321$ \\
\hline 72.3939 & 1.30543 & 8.51 & 0.1968 & $\mathrm{Sb}$ & 018 \\
\hline 73.2067 & 1.29293 & 5.95 & 0.1771 & $\mathrm{Sn}$ & 411 \\
\hline 79.4578 & 1.20618 & 6.76 & 0.1771 & $\mathrm{Sn}$ & 321 \\
\hline 89.3848 & 1.09617 & 4.78 & 0.1968 & $\mathrm{Sn}$ & 432 \\
\hline 95.5964 & 1.04071 & 3.69 & 0.1771 & $\mathrm{Sn}$ & 103 \\
\hline 97.5817 & 1.02391 & 1.68 & 0.576 & $\mathrm{Sn}$ & 521 \\
\hline
\end{tabular}

\begin{tabular}{|c|c|c|c|c|c|}
\hline \multicolumn{7}{|c|}{$\mathrm{Sn}_{79 .} \mathrm{Al}_{20}\left(\mathrm{TiO}_{2}\right)_{0.5}$} \\
\hline $2 \theta$ & $\mathrm{d} \AA$ & Int.\% & FWHM & Phase & hkl \\
\hline 30.378 & 2.94246 & 63.71 & 0.2362 & $\mathrm{Sn}$ & 200 \\
\hline 31.9942 & 2.79742 & 31.75 & 0.5987 & $\mathrm{Sn}$ & 101 \\
\hline 43.8632 & 2.06409 & 100 & 0.2165 & $\mathrm{Sn}$ & 220 \\
\hline 44.8424 & 2.02127 & 28.31 & 0.3542 & $\mathrm{Al}$ & 211 \\
\hline 55.3888 & 1.6588 & 12.76 & 0.3542 & $\mathrm{Sn}$ & 301 \\
\hline 62.57 & 1.48458 & 4.01 & 0.09 & $\mathrm{Sn}$ & 112 \\
\hline 63.8227 & 1.45843 & 25.99 & 0.1771 & $\mathrm{Sn}$ & 400 \\
\hline 64.6647 & 1.44146 & 12.5 & 0.3149 & $\mathrm{Al} / \mathrm{Sn}$ & $220 / 321$ \\
\hline 72.4103 & 1.30409 & 22.83 & 0.192 & $\mathrm{Sn}$ & 420 \\
\hline 73.158 & 1.29367 & 22.73 & 0.1771 & $\mathrm{Sn}$ & 411 \\
\hline 79.5208 & 1.20538 & 9.17 & 0.3149 & $\mathrm{Sn}$ & 321 \\
\hline 89.4656 & 1.09539 & 17.47 & 0.1771 & $\mathrm{Sn}$ & 431 \\
\hline 95.6294 & 1.04043 & 2.83 & 0.4723 & $\mathrm{Sn}$ & 103 \\
\hline 96.8422 & 1.02975 & 4.62 & 0.576 & $\mathrm{Sn}$ & 521 \\
\hline
\end{tabular}

\begin{tabular}{|c|c|c|c|c|c|}
\hline \multicolumn{7}{|c|}{$\mathrm{Sn}_{79} \mathrm{Al}_{20}\left(\mathrm{TiO}_{2}\right)_{1}$} \\
\hline $2 \theta$ & $\AA$ Int. & Int & FWHM & Phase & hkl \\
\hline 30.6095 & 2.92074 & 100 & 0.2755 & $\mathrm{Sn}$ & 200 \\
\hline 31.9936 & 2.79747 & 97.65 & 0.2362 & $\mathrm{Sn}$ & 101 \\
\hline 43.9771 & 2.05901 & 67.42 & 0.2165 & $\mathrm{Sn}$ & 220 \\
\hline 45.0299 & 2.01329 & 74.49 & 0.3346 & $\mathrm{Sn}$ & 211 \\
\hline 55.41 & 1.65822 & 11.24 & 0.09 & $\mathrm{Sn}$ & 301 \\
\hline 62.6126 & 1.48368 & 22.27 & 0.2362 & $\mathrm{Sn}$ & 112 \\
\hline 64.6723 & 1.44131 & 25.72 & 0.2362 & $\mathrm{Al} / \mathrm{Sn}$ & $220 / 321$ \\
\hline 72.67 & 1.30115 & 10.3 & 0.09 & $\mathrm{Sn}$ & 420 \\
\hline
\end{tabular}

Table 5b:- lattice parameters and crystal size of $\beta-\mathrm{Sn}$ in $\mathrm{Sn}_{80-\mathrm{x}} \mathrm{Al}_{20}\left(\mathrm{TiO}_{2}\right)_{\mathrm{x}}$ alloys

\begin{tabular}{|c|c|c|c|c|}
\hline Alloys & a $\AA$ & c $\AA$ & $\mathrm{V} \AA^{3}$ & $\tau \AA$ \\
\hline $\mathrm{Sn}_{80} \mathrm{Al}_{20}$ & 5.841 & 3.19 & 108.65 & 461.64 \\
\hline $\mathrm{Sn}_{79.5} \mathrm{Al}_{20}\left(\mathrm{TiO}_{2}\right)_{0.5}$ & 5.88 & 3.10 & 106.42 & 390.49 \\
\hline $\mathrm{Sn}_{79} \mathrm{Al}_{20}\left(\mathrm{TiO}_{2}\right)_{1}$ & 5.84 & 3.16 & 107.69 & 689.284 \\
\hline $\mathrm{Sn}_{78.5} \mathrm{Al}_{20}\left(\mathrm{TiO}_{2}\right)_{.1 .5}$ & 5.84 & 3.17 & 108.08 & 432.55 \\
\hline
\end{tabular}

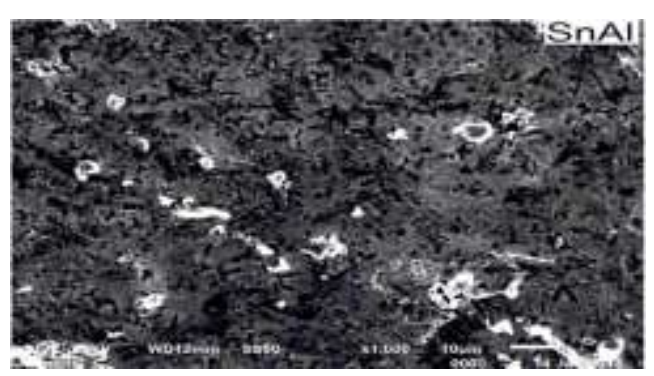



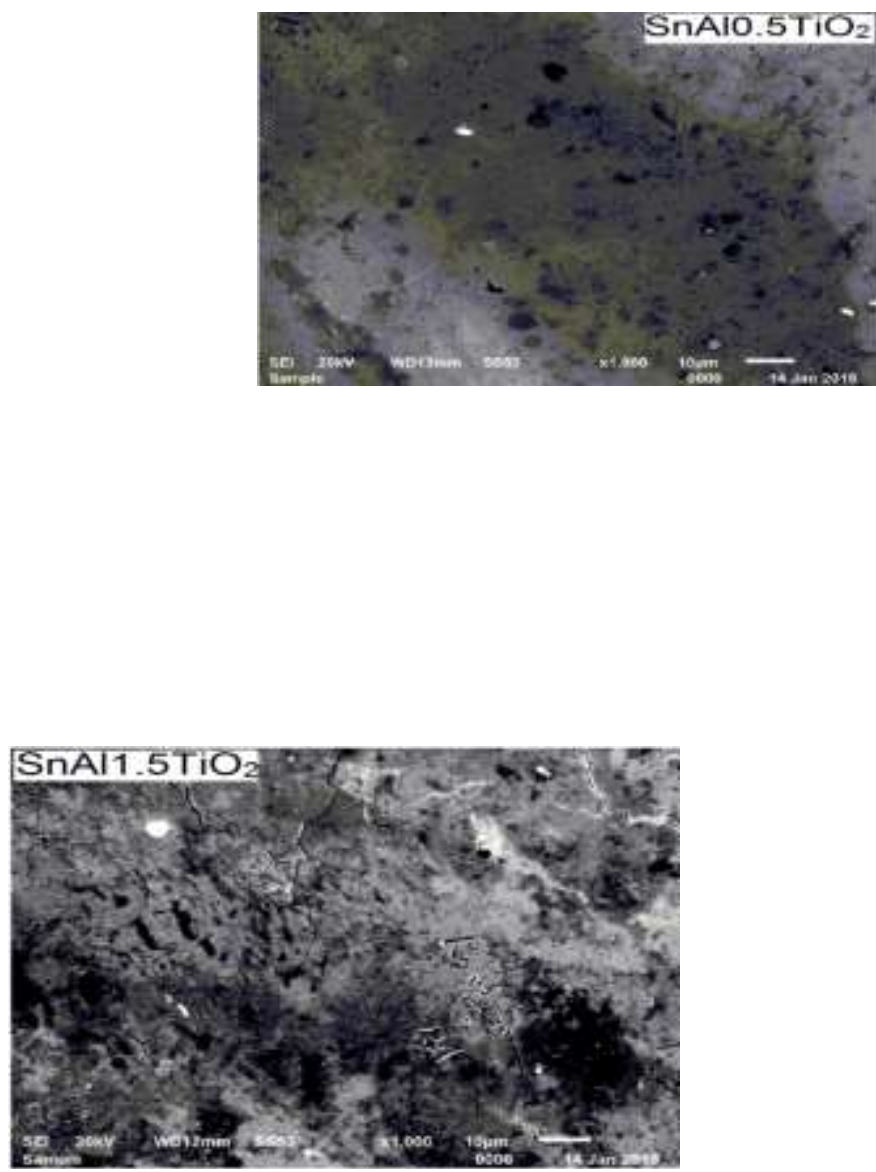

Figure 6:- $\mathrm{SEM}$ of $\mathrm{Sn}_{80-\mathrm{x}} \mathrm{Al}_{20}\left(\mathrm{TiO}_{2}\right)_{\mathrm{x}}$ alloys

Effect of adding $\mathrm{TiO}_{2}$ nanoparticles on mechanical properties of Sn- Al alloy

Elastic modului of $\mathrm{Sn}_{80-\mathrm{x}} \mathrm{Al}_{20}\left(\mathrm{TiO}_{2}\right)_{\mathrm{x}}$ alloys are listed in Table 6. Elastic modulus of $\mathrm{Sn}_{80} \mathrm{Al}_{20}$ alloy increased after adding different ratio from $\mathrm{TiO}_{2}$ nanoparticles. The $\mathrm{Sn}_{78.5} \mathrm{Al}_{20}\left(\mathrm{TiO}_{2}\right)_{1.5}$ alloy has highest elastic modulus.

The resonance curves $\mathrm{Sn}_{80-\mathrm{x}} \mathrm{Al}_{20}\left(\mathrm{TiO}_{2}\right)_{\mathrm{x}}$ alloys are shown in Figure 7. Calculated internal friction and thermal diffusivity of $\mathrm{Sn}_{80-}$ ${ }_{x} \mathrm{Al}_{20}\left(\mathrm{TiO}_{2}\right)_{\mathrm{x}}$ alloys are listed in Table 6. Internal friction of $\mathrm{Sn}_{80} \mathrm{Al}_{20}$ alloy increased after adding different ratio from $\mathrm{TiO}_{2}$ nanoparticles. The $\mathrm{Sn}_{78.5} \mathrm{Al}_{20}\left(\mathrm{TiO}_{2}\right)_{1.5}$ alloy has high internal friction value.

Table 6:- elastic modului, internal friction and thermal diffusivity of $\mathrm{Sn}_{80-\mathrm{x}} \mathrm{Al}_{20}\left(\mathrm{TiO}_{2}\right)_{\mathrm{x}}$ alloys

\begin{tabular}{|c|c|c|c|c|c|}
\hline Alloys & $\begin{array}{c}\mathrm{E} \\
\mathrm{GPa}\end{array}$ & $\begin{array}{c}\mu \\
\mathrm{GPa}\end{array}$ & $\begin{array}{c}\mathrm{B} \\
\mathrm{GPa}\end{array}$ & $\mathrm{Q}^{-1}$ & $\begin{array}{c}\mathrm{D}_{\text {th }} \times 10^{-} \\
8 \\
\left(\mathrm{~m}^{2} / \mathrm{sec}\right)\end{array}$ \\
\hline $\mathrm{Sn}_{80} \mathrm{Al}_{20}$ & 31.85 & 11.73 & 37.38 & 0.011 & 10.89 \\
\hline $\mathrm{Sn}_{79.5} \mathrm{Al}_{20}\left(\mathrm{TiO}_{2}\right)_{0.5}$ & 37.6 & 13.9 & 44 & 0.024 & 24.6 \\
\hline $\mathrm{Sn}_{79} \mathrm{Al}_{20}\left(\mathrm{TiO}_{2}\right)_{1}$ & 38.9 & 14.3 & 45.4 & 0.0227 & 60.97 \\
\hline $\mathrm{Sn}_{78.5} \mathrm{Al}_{20}\left(\mathrm{TiO}_{2}\right)_{1.5}$ & 40.8 & 15 & 47.4 & 0.0228 & 9.60 \\
\hline
\end{tabular}

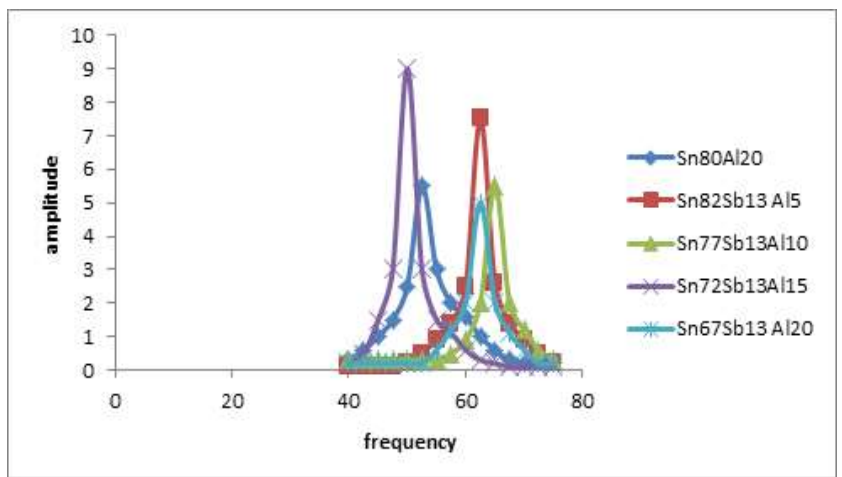

Figure 7:- resonance curves of $\mathrm{Sn}_{80-\mathrm{x}} \mathrm{Al}_{20}\left(\mathrm{TiO}_{2}\right)_{\mathrm{x}}$ alloys

Vickers hardness of $\mathrm{Sn}_{80-\mathrm{x}} \mathrm{Al}_{20}\left(\mathrm{TiO}_{2}\right)_{\mathrm{x}}$ alloys at 10 gram force and indentation time $5 \mathrm{sec}$ are shown in Table 7. The minimum shear stress $\left(\tau_{\mathrm{m}}\right)$ value of $\mathrm{Sn}_{80-\mathrm{x}} \mathrm{Al}_{20}\left(\mathrm{TiO}_{2}\right)_{\mathrm{x}}$ alloys was calculated then listed in Table 7. Little variation occurred in Vickers hardness of $\mathrm{Sn}_{80} \mathrm{Al}_{20}$ alloy after adding $\mathrm{TiO}_{2}$ nanoparticles.

Table 7:- Vickers hardness and minimum shear stress of $\mathrm{Sn}_{80-\mathrm{x}} \mathrm{Al}_{20}\left(\mathrm{TiO}_{2}\right)_{\mathrm{x}}$ alloys

\begin{tabular}{|c|c|c|}
\hline Alloys & $\mathrm{H}_{\mathrm{v}} \mathrm{kg} / \mathrm{mm}^{2}$ & $\mu_{\mathrm{n}} \mathrm{kg} / \mathrm{mm}^{2}$ \\
\hline $\mathrm{Sn}_{80} \mathrm{Al}_{20}$ & $36.43 \pm 2.7$ & 14.33 \\
\hline $\mathrm{Sn}_{79.5} \mathrm{Al}_{20}\left(\mathrm{TiO}_{2}\right)_{0.5}$ & $35.77 \pm 1.9$ & 11.80 \\
\hline $\mathrm{Sn}_{79} \mathrm{Al}_{20}\left(\mathrm{TiO}_{2}\right)_{1}$ & $37.88 \pm 2.2$ & 12.50 \\
\hline $\mathrm{Sn}_{78.5} \mathrm{Al}_{20}\left(\mathrm{TiO}_{2}\right)_{1.5}$ & $38.92 \pm 3.1$ & 12.84 \\
\hline
\end{tabular}

Effect of adding $\mathrm{TiO}_{2}$ on thermal properties and electrical resistivity of $\mathrm{Sn}-\mathrm{Al}$ alloy

Figure 8 shows DSC thermographs for $\mathrm{Sn}_{80-\mathrm{x}} \mathrm{Al}_{20}\left(\mathrm{TiO}_{2}\right)_{\mathrm{x}}$ alloys. Little variation occurred in exothermal peak of $\mathrm{Sn}_{80} \mathrm{Al}_{20}$ alloy. The melting temperature and other thermal properties of $\mathrm{Sn}_{80}$ ${ }_{x} \mathrm{Al}_{20}\left(\mathrm{TiO}_{2}\right)_{\mathrm{x}}$ alloys are listed in Table 8. Melting temperature of $\mathrm{Sn}_{80} \mathrm{Al}_{20}$ alloy increased after adding $\mathrm{TiO}_{2}$ nanoparticles.

Electrical resistivity and calculated thermal conductivities of $\mathrm{Sn}_{80-\mathrm{x}} \mathrm{Al}_{20}\left(\mathrm{TiO}_{2}\right)_{\mathrm{x}}$ alloys are listed in Table 8. Electrical resistivity of $\mathrm{Sn}_{80} \mathrm{Al}_{20}$ alloy varied after adding $\mathrm{TiO}_{2}$ nanoparticles. That is because $\mathrm{TiO}_{2}$ nanoparticles dissolved in the Sn matrix playing as scattering center for conduction electrons caused a change in $\mathrm{Sn}$ matrix.

Table 8:- electric resistivity and other thermal properties of $\mathrm{Sn}_{80-\mathrm{x}} \mathrm{Al}_{20}\left(\mathrm{TiO}_{2}\right)_{\mathrm{x}}$ alloys

\begin{tabular}{|c|c|c|c|c|c|}
\hline Alloys & $\begin{array}{l}\rho \times 10^{-6} \\
\Omega . \mathrm{cm}\end{array}$ & $\begin{array}{l}\mathrm{K} \mathrm{W} \\
\mathrm{m}^{-1} \mathrm{~K}^{-1}\end{array}$ & $\begin{array}{l}\mathrm{C}_{\mathrm{p}} \times 10^{3} \\
\mathrm{~J} / \mathrm{g} .{ }^{\circ} \mathrm{C}\end{array}$ & $\begin{array}{l}\Delta \mathrm{S} \\
\mathrm{J} / \mathrm{g} .{ }^{\circ} \mathrm{C}\end{array}$ & $\begin{array}{l}\text { Melting } \\
\text { point }{ }^{\circ} \mathrm{C}\end{array}$ \\
\hline $\mathrm{Sn}_{80} \mathrm{Al}_{20}$ & 67.3 & 1.21 & 0.723 & 0.122 & 227.03 \\
\hline
\end{tabular}


International Journal of Science and Engineering Applications

Volume 4 Issue 2, 2015, ISSN-2319-7560 (Online)

\begin{tabular}{|c|c|c|c|c|c|}
\hline $\mathrm{Sn}_{79.5} \mathrm{Al}_{20}\left(\mathrm{TiO}_{2}\right)_{0.5}$ & 137.30 & 2.1 & 3.74 & 0.198 & 228.22 \\
\hline $\mathrm{Sn}_{79} \mathrm{Al}_{20}\left(\mathrm{TiO}_{2}\right)_{1}$ & 77.76 & 2.31 & 2.85 & 0.190 & 228.92 \\
\hline $\mathrm{Sn}_{78.5} \mathrm{Al}_{20}\left(\mathrm{TiO}_{2}\right)_{.1 .5}$ & 68.40 & 3.27 & 3.34 & 0.2 & 229.63 \\
\hline
\end{tabular}

Figure 8:- $\mathrm{DSC}$ of $\mathrm{Sn}_{80-\mathrm{x}} \mathrm{Al}_{20}\left(\mathrm{TiO}_{2}\right)_{\mathrm{x}}$ alloys
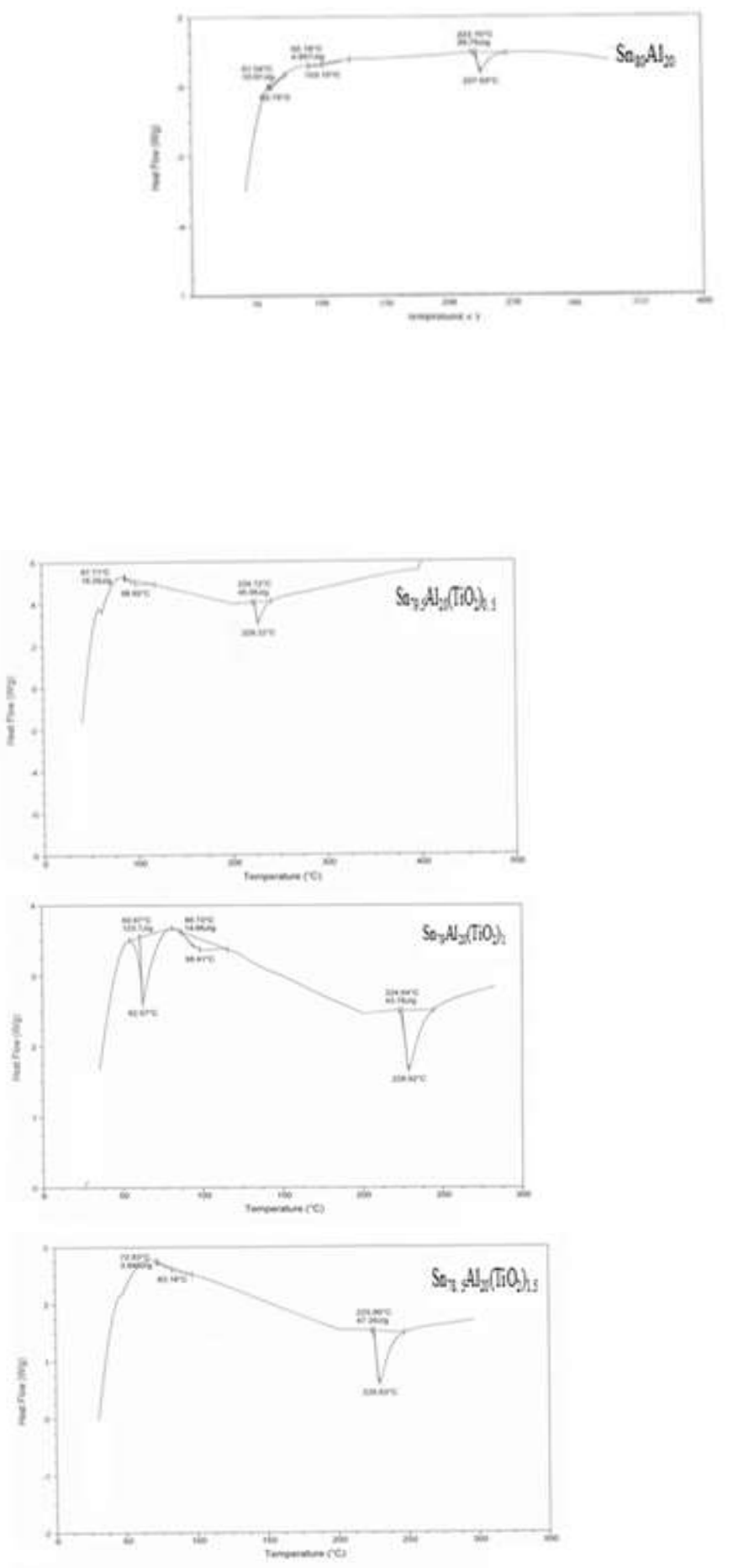

\section{Conclusion}

Structure of $\mathrm{Sn}_{87} \mathrm{Sb}_{10} \mathrm{~Pb}_{3}$ and $\mathrm{Sn}_{80} \mathrm{Al}_{20}$ alloys changed after adding $\mathrm{TiO}_{2}$ nanoparticles. Elastic modulus and Vickers hardness of $\mathrm{Sn}_{87} \mathrm{Sb}_{10} \mathrm{~Pb}_{3}$ and $\mathrm{Sn}_{80} \mathrm{Al}_{20}$ alloys increased after adding $\mathrm{TiO}_{2}$ nanoparticles. Internal friction and mmelting temperature of $\mathrm{Sn}_{87} \mathrm{Sb}_{10} \mathrm{~Pb}_{3}$ alloy decreased but internal friction and melting temperature of $\mathrm{Sn}_{80} \mathrm{Al}_{20}$ alloy increased after adding $\mathrm{TiO}_{2}$ nanoparticles. The $\mathrm{Sn}_{85.5} \mathrm{Sb}_{10} \mathrm{~Pb}_{3}\left(\mathrm{Ti}_{2} \mathrm{O}\right)_{1.5}$ alloy has beast properties for automotive industry. Also $\mathrm{Sn}_{78.5} \mathrm{Al}_{20}\left(\mathrm{Ti}_{2} \mathrm{O}\right)_{1.5}$ alloy has beast properties for marine applications.

\section{References}

[1] Forrester P. G, Met. Rev. 5 (1960) 507

[2] Pratt G. C, Int. Met. Rev. 18 (1973) 62

[3] Forrester P. G, Curr. Engg. Pract 3 (1961) 4

[4] Tegart W. I. M, Elements of mechanical metallurgy (New York: The MacMillan Co.) (1966) 91

[5] Desaki T, Goto Y, Kamiya S, JSAE Rev. 21 (2000) 321

[6] Lepper K, James M, Chashechkina J, Rigney D. A, Wear 46 (1997) 203

[7] Perrin C, S. Harris, McCartney D. G, Syngellakis S, Reed P. A, in: J.F. Morton, B.C. Muddle (Eds.), Mater. Forum 28 (2004) 1371

[8] Kong C. J, Brown P. D, S, Harris J, McCartney D. G, Mater. Sci. Eng. A 403 (2005) 205

[9] Ning X. J, Jang J. H, Kim H. J, Li C. J, C. Lee, Surf. Coat. Technol. 202 (2008) 1681

[10] Ning X. J, Kim J. H, Kim H. J, Lee C, Applied Surface Science 255 (2009) 3933

[11] Pathak J. P and Mohan S, Bull. Mater. Sci. 26:3 (2003) 315

[12] Kama M, El-Bediwi A and El-Shobaki M, Radia. Eff. Def. Sol. 161 (2006) 549

[13] Bora M. O, Coban O, Sinmazcelik T, Gunay V, Zeren M, Mater. Design 31 (2010) 2707

[14] El- Bediwi A. B, El Said Gouda, Kamal M, AMSE, 65, n 1 , Modeling C- 2004

[15] Kamal M, Abdel-Salam A, Pieri J. C, J. Mater. Sci. 19 (1984) 3880

[16] El-Bediwi A, Lashin A, Mossa M, Kamal M, Mater. Sci. Eng. A 528 (2011) 3568

[17] E Schreiber, Anderson O. L and Soga N, Elastic constant and their measurements, McGraw-Hill, New York, (1973) 82

[18] Timoshenko S and Goddier J. N, "Theory of elasticity, $2^{\text {nd }}$ Ed", McGraw-Hill, New York, (1951) 277

[19] Nuttall K, J. Inst. Met. 99 (1971) 266 